\title{
La question de l'altruisme dans la première réception russe de Nietzsche (Preobrajenski, Lopatine, Grot, Astafiev)
}

Antoine Muller

\section{(2) OpenEdition Journals \\ Édition électronique \\ URL : https://journals.openedition.org/cpuc/943 \\ DOI : $10.4000 /$ cpuc. 943 \\ ISSN : 2677-6529 \\ Éditeur}

Presses universitaires de Caen

\section{Édition imprimée}

Date de publication : 31 octobre 2011

Pagination : 11-45

ISBN : 978-2-84133-385-1

ISSN : 1282-6545

Référence électronique

Antoine Muller, « La question de l'altruisme dans la première réception russe de Nietzsche

(Preobrajenski, Lopatine, Grot, Astafiev) », Cahiers de philosophie de l'université de Caen [En ligne], 48 2011, mis en ligne le 01 septembre 2020, consulté le 10 février 2023. URL : http:// journals.openedition.org/cpuc/943; DOI : https://doi.org/10.4000/cpuc.943

\section{(c) (7) (8)}

Creative Commons - Attribution - Pas d'Utilisation Commerciale 4.0 International - CC BY-NC 4.0 https://creativecommons.org/licenses/by-nc/4.0/ 


\section{La question de l'altruisme dans la première réception russe de Nietzsche (Preobrajenski, Lopatine, Grot, Astafiev)}

\footnotetext{
T E PREMIER DÉBAT RUSSE consacré à part entière à Nietzsche a lieu dans les pages de la revue Voprosy filosofii i psikhologii (Questions de philosophie et de psychologie) ${ }^{1}$. Le débat prend la forme d'un exposé plutôt enthousiaste, dans le numéro 15 (1892), auquel répondent dans le numéro suivant (1893) trois réquisitoires diversement orientés, mais unanimement critiques. Il porte sur la question morale de l'altruisme.

La publication du premier article, «Friedrich Nietzsche, une critique de la morale de l'altruisme ${ }^{2}$, dû à Vassili Petrovitch Preobrajenski ${ }^{3}$, est accompagnée d'une note de la rédaction dont le ton contraste fortement avec celui de l'article lui-même:
}

La rédaction se décide à publier pour les lecteurs russes l'exposé de la doctrine morale de Fr. Nietzsche, révoltante par ses conclusions finales, à cette fin de montrer quels phénomènes étranges et maladifs frappent actuellement une certaine tendance de la culture d'Europe occidentale. Friedrich Nietzsche, écrivain et penseur talentueux, qui ne manque ni d'éclat ni de finesse, aveuglé par sa haine de la religion, du christianisme et de Dieu lui-même, prêche

1. L'ensemble des quatre textes qui font l'objet de cette étude ont été réédités dans le volume Ницше: pro et contra, Ю. В. Синеокая (ред.), СПб., РХГИ, 2001 (Preobrajenski : p. 35-69; Lopatine: p. 70-74; Grot: p. 75-94; Astafiev: p. 95-112). Sauf indication contraire, les numéros de page donnés dans le corps du texte de cette étude renvoient à cet ouvrage.

2. «Фридрих Ницше, Критика морали альтруизма ", paru pour la première fois dans: Вопросы философии и психологии, № 15, 1892, р. 115-160.

3. Василий Петрович Преображенский (1864-1900), a enseigné un temps à la faculté d'histoire et de philologie de l'université de Moscou, et soutenu une thèse sur Spencer, avant de se spécialiser dans l'édition et la traduction, en se consacrant notamment à Leibniz et Spinoza. Son article sur Nietzsche a fait date et lui a valu de voir son nom associé à celui du penseur allemand, à tel point que $\mathrm{P}$. Boborykine s'en servit de modèle pour le personnage de Kostritsyne, héros de Перевал (Le passage), qui professe des théories clairement inspirées d'une certaine vulgate nietzschéenne. 
cyniquement une indulgence complète pour le crime, pour la débauche la plus effrayante et pour la déchéance morale au nom d'un idéal de perfectionnement de représentants isolés de l'espèce humaine, en outre la masse de l'humanité est considérée de façon sacrilège comme un piédestal pour l'élévation de «génies » dévergondés, et que ne retiennent aucune limite de la loi et de la moralité, dans le genre de Nietzsche lui-même. Et quelle leçon grande et instructive fournit le destin de cet orgueilleux infortuné, qui s'est retrouvé dans une maison d'aliénés par suite de son idee fixe ${ }^{4}$, qu'il était le Créateur du monde. Cette punition, grande et méritée d'un malheureux athée, qui s'imaginait être un dieu, suscite un effroi véritable. On ne pouvait pas dans une revue philosophique passer sous silence un fait aussi important et édifiant dans l'histoire des aberrations philosophiques contemporaines. Dans le prochain tome, nous publierons une analyse plus détaillée de l'aspect philosophique de l'enseignement de Nietzsche, par quelques collaborateurs du journal (Lopatine, Astafiev et Grot).

La rédaction. (p. 1009)

Plusieurs éléments attirent l'attention. Tout d'abord la seule concession faite à Nietzsche concerne son style, ses qualités littéraires. C'est un point qui d'une façon ou d'une autre est presque systématiquement signalé par les commentateurs russes, quelle que soit leur évaluation du contenu doctrinal nietzschéen. En second lieu, trois couples d'adjectifs de sens voisin marquent les points sur lesquels la rédaction a jugé bon d'insister: nestchastny / zlopoloutchny (infortuné / malheureux), veliki / kroupny (grand / important), pooutchitel'ny / nazidatel'ny (instructif / édifiant). Les jugements sans appel portés contre Nietzsche (athée, orgueilleux, cynique) sont contrebalancés par le postulat d'une sorte d'irresponsabilité morale, qui découle d'un aveuglement religieux. Le contenu de la doctrine nietzschéenne est résumé par deux idées centrales : dévergondage moral, et corrélativement, idéal inégalitaire. Mais surtout, l'ensemble est présenté comme découlant logiquement de l'athéisme de l'auteur, qui l'amène à prendre en pensée la place de Dieu et cause finalement sa folie. Ce qui rend Nietzsche incontournable, ce n'est donc pas du tout sa pensée même (qualifiée d' «aberration»), c'est son « destin», qui en est inséparable parce qu'il lui donne tout son sens, à titre de «leçon» édifiante, et l'objectif avoué de l'article est d'éveiller une horreur sacrée, un «effroi véritable».

Tout se passe donc comme si l'on prévenait pour guérir, comme si, du moins en apparence, on laissait filtrer des éléments "occidentaux» afin d'éviter un mal plus grand. La parution de l'article est soumise à une double caution: il est encadré par cet avertissement introductif, et

4. En français (sans accent) dans le texte. 
on annonce d'emblée pour le numéro suivant trois réponses à «l'aspect philosophique» de la doctrine nietzschéenne.

\section{Preobrajenski}

On s'attendrait après pareille introduction à ce que le premier article condamne Nietzsche sans appel, ou du moins à ce que ses idées soient présentées sous un jour si manifestement abominable qu'elles se passent de commentaires. Or l'exposé de Preobrajenski contraste nettement avec la note éditoriale. Ce qui frappe en premier lieu, c'est son ordonnancement rigoureux - en quinze points -, sa volonté évidente de prendre au sérieux la pensée de Nietzsche, et une admiration qui transparaît malgré la volonté affichée de s'en tenir à un exposé. On trouve dans le texte une proportion très importante de citations, souvent longues, dont seule une petite partie est signalée (les citations et paraphrases représentent environ deux tiers du texte, et même davantage si l'on exclut l'introduction et la conclusion qui n'en comportent pas). Le procédé le plus récurrent consiste à ne mettre qu'une partie de la citation entre guillemets. Compte tenu de la double censure qui avait encore cours à cette date, il est possible que Preobrajenski utilise son article pour publier la plus grande quantité possible de traductions, ce qui s'accorde tout à fait avec la conviction qu'il professe à la fin de la première partie de son exposé:

Nietzsche appartient précisément à cette catégorie de penseurs-artistes, qu'il est absolument nécessaire de connaître dans leurs propres œuvres pour les connaître sous leur aspect véritable - de la même façon qu'aucun exposé n'est à même de donner une idée véritable du génie artistique et philosophique de Platon à celui qui n’a pas fait connaissance avec ses œuvres originales. (p. 36-37)

La recension de Preobrajenski s’ingénie à rapprocher une grande quantité de passages en provenance de presque toute l'œuvre, et témoigne d'une familiarité profonde avec Nietzsche. Il fait appel majoritairement à ce qu'il appelle "la meilleure période de Nietzsche» (Humain trop humain, Aurore, Le gai savoir) dont il n'hésite pas à considérer les œuvres comme «les meilleurs spécimens de la prose allemande, sans exclure Goethe et Schopenhauer». Un coup d'œil sur la provenance des textes qu'il utilise, en les citant ou sans les mentionner, confirme en gros cette évaluation. Vingt-quatre paragraphes d'Aurore sont employés ${ }^{5}$, dix-sept du Gai savoir ${ }^{6}$, sept d'Humain trop humain

5. Aurore, $\$ 13,76,80,106,107,108,132,133,134,137,139,144,146,148,164,173,174,187,202$, $334,453,516,517,529$. Cet inventaire et ceux qui suivent prennent en compte les textes dont Preobrajenski ne donne pas la référence, il est possible que certains m’aient échappé.

6. Le gai savoir, $\$ 1,4,7,19,21,62,108,116,270,283,289,290,294,338,345,357,380$. 
(plus deux du Voyageur et son ombre) ${ }^{7}$. Mais Preobrajenski fait également un usage abondant de Par-delà bien et mal (quinze textes, une mention à la dernière section en général ${ }^{8}$, La généalogie de la morale (six textes, deux mentions générales) ${ }^{9}$ et Le crépuscule des idoles (six textes, une mention générale $)^{10}$. Enfin les Considérations inactuelles font l'objet de cinq utilisations, dont une mention générale, deux à la Seconde, une à la Troisième et une à la Quatrième ${ }^{11}$. En revanche seuls deux textes d'Ainsi parlait Zarathoustra sont cités, dont l'un constitue un doublon avec le dernier texte du Crépuscule des idoles. Quant à La naissance de la tragédie, il n'y est pas fait mention dans le texte. Or ces deux derniers ouvrages sont précisément ceux que les textes russes postérieurs mentionnent le plus volontiers ${ }^{12}$. On peut voir deux raisons à ce fait. D'abord la problématique choisie par Preobrajenski est morale, et non esthétique. Ensuite ces deux livres ont chacun à leur manière un statut particulier dans l'œuvre nietzschéenne, et fonctionnent davantage comme des ensembles clos que les recueils d'aphorismes. On note en particulier l'absence de la section du Zarathoustra, «De l'amour du prochain». C'est que le parti pris de la démarche de Preobrajenski est de reconstituer un ensemble cohérent à partir des textes épars de Nietzsche. Non pas d'ailleurs qu'il considère la forme aphoristique comme un défaut, puisqu'il la qualifie de «style littéraire original, amené par Nietzsche à un degré suprême de perfection, s'il ne l'a pas même créé le premier sous cette forme en particulier».

Chacun de ces aphorismes [...] présente un groupe particulier de pensées, étroitement lié et intérieurement achevé, exprimé sous une forme concise, entière et artistiquement achevée [...]. Ils frappent par la force et la souplesse de leur langue, par la maîtrise étonnante dans la transmission des nuances de pensée et des humeurs les plus insaisissables et capricieuses, et par l'élégance de leur forme, tantôt plastique et comme frappée ${ }^{13}$, tantôt sonore et expressive comme une musique. (p. 36)

7. Humain trop humain, préface, $\$ 6,47,49,57,103,233,321$; Le voyageur et son ombre, $\$ 95,350$.

8. Par-delà bien et mal, $\$ 23,30,44,186,201,203,212,220,225,228,257,258,268,296$.

9. La généalogie de la morale, I, $\$ 11,12,17$ (note); II, $\$ 24$; III, $\$ 14,28$. Une mention aux «parties II et III».

10. Le crépuscule des idoles, "Maximes et flèches», $\$ 12$, 26 ; «Incursions d'un inactuel », $\$ 37$, $38,45,48$, dernier $\$$ (le marteau parle).

11. Seconde considération inactuelle, chap. IX (deux fois); Troisième considération inactuelle, chap. I; Quatrième considération inactuelle, chap. II.

12. On songe notamment à la fortune du Zarathoustra chez Chestov, chez Biély et les autres symbolistes, ainsi qu'aux lectures de La naissance de la tragédie centrées tantôt sur le dionysiaque, plus ou moins assimilé au thème christique (Viatcheslav Ivanov), tantôt sur l’apollinien (Ossip Mandelstam et les acméistes).

13. «Вычеканенной». 
Néanmoins dans la conclusion de son étude, Preobrajenski écrit:

Nous nous sommes efforcé avant tout de montrer et rendre sinon convaincante, du moins intelligible, cette logique intérieure, qui unit les étapes successives, qui parfois ont l'air de bonds, du cours des pensées de Nietzsche, et lie dans un certain ensemble ce qu'une main généreuse, mais capricieuse, a jeté dans des lieux divers et dans différents livres. (p. 68)

Affirmation qui entre en tension avec ce qui était dit plus haut d'un texte de Nietzsche auquel Preobrajenski se référait sans le citer, et dont il donnait précisément Nietzsche comme le premier contre-exemple:

Nietzsche empreint d'un regret visible, dit quelque part qu'il est impossible de trouver un seul homme en Allemagne qui se soit déterminé à travailler à deux petites pages de prose, comme à une statue: c'est justement de cette manière-là que Nietzsche travaillait à ses aphorismes. (p. 36-37)

Mais cette contradiction apparente est liée en grande partie aux objectifs délimités de l'exposé. Preobrajenski prend d'ailleurs acte d'emblée des risques de l'entreprise:

Une systématisation des vues de Nietzsche amènera souvent à extraire de force des pensées isolées de ce groupe entier et comme moulé dans lequel elles rentrent - de l'aphorisme. (p. 38)

Cela n'enlève rien au fait que la fidélité de Preobrajenski au texte original qu'il traduit est souvent toute relative. À certains endroits, on peut soupçonner que la censure ou l'autocensure ont joué un rôle. En étudiant de près les différences avec l'original allemand ou les oublis de la paraphrase russe, certaines hypothèses peuvent être présentées.

Ainsi à la fin de son troisième point, Preobrajenski cite presque en entier le paragraphe 107 d'Aurore. La référence est précisée et le texte de Nietzsche traduit quasiment mot pour mot, mais les guillemets ne sont pas utilisés, et en plein paragraphe, deux passages de quelques lignes manquent. On est en droit de supposer que ce n'est pas un hasard si ce sont précisément les passages qui évoquent ce que Nietzsche appelle les «tenants de la morale autoritaire». Voici ce que l'on obtient si l'on restitue, hors crochets, les passages oblitérés:

14. Cf. Le voyageur et son ombre, $\$ 95$ : «der Deutsche nur die improvisierte Prosa kennt und von einer anderen gar keinen Begriff hat [...] an einer Seite Prosa wie an einer Bildsäule arbeiten? - es ist ihm, also ob man ihm etwas aus dem Fabelland vorerzählte». 
[Cette autorité de la morale entrave la pensée en des matières où il pourrait être dangereux de penser $f a u x]$ - : c'est ainsi qu'elle a coutume de se justifier devant ses accusateurs. Faux : cela veut dire ici «dangereux», - mais dangereux pour qui? Habituellement ce n'est à vrai dire pas le danger couru par l'auteur de l'action que les tenants de la morale autoritaire ont en vue, mais leur propre danger, leur perte éventuelle de puissance et de prestige dès l'instant où l'on reconnaîtrait à tous le droit d'agir arbitrairement et follement, [selon leur propre raison, restreinte ou étendue] : en ce qui les concerne, ils n'hésitent pas à user de ce droit à l'arbitraire et à la folie, - ils ordonnent, même dans les cas où les questions «Comment dois-je agir Dans quel but dois-je agir?» peuvent à peine et fort difficilement recevoir une réponse. (p. 42) ${ }^{15}$

Sans aucun doute, l'une des logiques auxquelles obéit Preobrajenski consiste à simplifier les ramifications du texte allemand, en supprimant la prosopopée de "l'autorité de la morale». Mais en faisant disparaître la suite, il évite prudemment de déterminer trop concrètement quelles formes matérielles, institutionnelles, peut prendre cette autorité, et atténue le conflit de forces et d'intérêts décrit par Nietzsche: aussitôt que «penser faux» est mis en équation par l'ellipse avec «selon leur propre raison, restreinte ou étendue», on se rapproche du modèle classique qui attribue l'erreur au jugement «subjectif», insuffisamment méthodique.

Second exemple: à la fin de son huitième point, consacré à la «morale du troupeau» (p. 56), Preobrajenski traduit la seconde moitié du paragraphe 203 de Par-delà bien et mal. On relève un certain nombre d'approximations : «Es gibt wenig» («il y a peu») est traduit «niet» («il n'y a pas»), plusieurs verbes au passé sont traduits au présent, les guillemets autour de "L'homme» sont supprimés, ainsi que plusieurs expressions de-ci de-là. Sans doute s'agissait-il de couper court à des nuances qui auraient compliqué l'accès à l'essentiel - et il n'est pas certain que l'auteur ait ici saisi les enjeux qui font de certaines de ces nuances davantage que de simples coquetteries formelles. Mais à nouveau, un autre type de retouche doit être distingué des exemples précédents: l'article est très précautionneux partout où Nietzsche emploie le mot Dieu et souvent lorsque l'élément religieux en général se trouve remis en cause. Ainsi, lorsque Nietzsche décrit la «contingence monstrueuse» du destin de l'homme, il qualifie ce dernier de «jeu auquel nulle main n'a participé, et pas même le "doigt de Dieu"!", formule qui disparaît en russe. De la même façon, une formule du Gai savoir (\$108) qui se rapportait directement à la mort de Dieu est modifiée (p. 57) : Preobrajenski traduit d'assez près le début, qui traite de la mort de Bouddha

15. F. Nietzsche, Aurore, Paris, Gallimard, 1970 (trad. par J. Hervier). 
(entre guillemets, mais sans référence), puis fausse compagnie au texte au moment précis où Nietzsche écrit «Dieu est mort», la fin de la paraphrase détournant l'assaut contre les «valeurs morales» sur un plan assez abstrait, et surtout il est question des "vieilles idoles»: en accordant la cible au pluriel, Preobrajenski garde l'apparence d'une sorte d'orthodoxie à l'égard du thème vétérotestamentaire qu'est le combat contre les vieilles idoles. Quelques lignes plus bas, une citation empruntée à La généalogie de la morale (II, \$24) est introduite par un ajout, tronquée de manière abrupte, et subit une petite entorse en son milieu:

Un jour ${ }^{16}$, dit Nietzsche, viendra cet homme de l'avenir qui sera le rédempteur nous délivrant aussi bien de l'idéal ayant existé jusqu'à présent que de ce qu'il a fait pousser, du grand dégoût, de la volonté de néant, du nihilisme, ce coup de cloche de midi et de la grande décision qui libérera à nouveau la volonté, qui restituera à la terre son but et à l'homme son espérance! (p. 57 $)^{17}$

Tout d'abord, l'amorce associée au point d'exclamation final donne à la phrase l'apparence d'une promesse. Ensuite, Preobrajenski coupe «cet antéchrist et cet antinihiliste, ce vainqueur de Dieu et du néant - il faudra qu'il vienne un jour...». L'équation Dieu = néant était certes trop tendancieuse pour être conservée. Mais si l'on examine cette phrase en la rapprochant d'une autre correction apportée par le traducteur, une autre dimension du texte auquel il n'a pas été sensible ou qui lui paraissait trop scandaleuse apparaît: celle de la nécessité du processus, marquée par le verbe allemand müssen. Là où Preobrajenski traduit «ce qu'il a fait pousser», Nietzsche écrivait en effet, en italique: «dem, was aus ihm wachsen musste»: «ce qu’il devait nécessairement faire pousser». Le traducteur enlève ou aplanit donc ici les deux bouts de phrase que Nietzsche avait cru bon de mettre en italique! Sa prudence à l'égard des motifs religieux relève-t-elle d'une auto-censure préventive, ou d'un consensus avec la rédaction, et la note de la rédaction (qui met clairement l'accent sur l'errance religieuse de Nietzsche) est-elle apparue conjointement à la censure des passages en question, ou bien malgré une atténuation antérieure des textes? Il est bien difficile de trancher, mais il faut sans doute se garder de deux écueils: voir en Preobrajenski un nietzschéen inconditionnel confronté à un front absolument anti-nietzschéen; appliquer à son travail des critères de scientificité et de compréhension qui ne tiendraient pas compte de ses objectifs réels - introduire une pensée et non établir un texte.

16. «Когда же».

17. F. Nietzsche, La généalogie de la morale, Paris, LGF, 2000 (trad. par P. Wotling), II, $\$ 24$ (traduction modifiée en fonction de celle de Preobrajenski). 
Comme on l'a exposé, la plupart des imprécisions que l'on repère en comparant le texte russe à l'original ne paraissent pas liées à la censure. Certaines formules de Nietzsche dont on peut penser qu'elles étaient les plus surprenantes ou les plus atypiques (l'idée d' «élevage» ou celle de «tentative ${ }^{18}$ ) sont supprimées - mais il est évident que ce qui rend un lecteur contemporain aussi sensible à ces " oublis", ce sont les commentaires qui se sont attachés depuis à soutenir l'importance de ces thèmes. Preobrajenski ôte également certaines interventions de Nietzsche dans son texte (adresses au lecteur...), et nombre d'images comme celle de la «plante». Il arrive que l'on ne sache pas très bien si les modifications sont dues à la complexité des schémas affectifs (et des tournures verbales correspondantes) ou à une certaine pudeur soucieuse de trouver des variations moins provocantes: quand Nietzsche écrit: «Das liebende Mädchen wünscht, dass sie die hingebende Treue ihrer Liebe an der Untreue des Geliebten bewähren könne» ${ }^{19}$, au lieu de dire qu'une jeune fille souhaite mettre sa fidélité à l'épreuve de l'infidélité de l'aimé, Preobrajenski traduit qu'elle «désire conserver la fidélité trahie de son amour même en cas d'infidélité de son bien-aimé » (p. 48-49), ce qui affaiblit le sens original (et fait jouer un tout autre rôle au personnage féminin!).

La difficulté vient donc, pour résumer, de ce qu'il n'est pas toujours évident de déceler si les transformations formelles de détail sont voulues, et si elles constituent des simplifications, ou bien de réelles concessions du traducteur. Preobrajenski change fréquemment le nombre des noms, omet la plupart des points d'exclamation (peut-être pour ne pas avoir l'air de prendre à son compte l'emphase nietzschéenne lorsqu'il traduit sans guillemets), ne retranscrit pas toujours les italiques et les guillemets du texte. Il coupe de temps à autre les conclusions de passages qu'il a cités in extenso et surtout efface souvent les réserves prudentes et les nuances par lesquelles Nietzsche détermine le point de vue qui formule tel ou tel jugement, par exemple en introduisant brusquement avec des formules comme «il faut» («noujno») un texte dont il résume de façon cavalière le début en une formule discutable, ou en remplaçant une interrogative négative par une affirmative (p. 65) ${ }^{20}$, mais aussi en faisant disparaître les occurrences du pronom personnel «nous», et plusieurs éléments qui multiplient le nombre de "voix» du texte et manifestent le fait qu'à travers les personnes, ce sont tels et tels affects qui se font entendre à un moment donné. Ainsi au paragraphe 133 d'Aurore:

18. «Züchten» et «Versuch».

19. Humain trop humain, $\$ 57$.

20. Par-delà bien et mal, $\$ 225$. 
Pourquoi saute-t-on à l'eau pour secourir quelqu'un que l'on y a vu tomber, bien que l'on n'ait pour lui aucune sympathie particulière? Par compassion: on ne pense plus qu'à autrui, - dit l'irréflexion. (p. 44)

Preobrajenski supprime l'incise finale, ce qu'il compense par l'ajout de guillemets à «compassion ». Par ailleurs, la traduction rend fréquemment un adjectif ou un verbe allemands par deux termes en russe. "Macht» est généralement rendu "vlast'» ("pouvoir»), comme chez la quasi-totalité des commentateurs russes jusqu'à aujourd'hui, mais lorsque Preobrajenski utilise le terme «mochtch» ("puissance») à la place, il le renforce avec «obladanie» («appropriation»). Il remplace de temps à autre un terme par un autre ( «esprits» par «hommes», "modèles» ou ailleurs «lois» par «idéaux»), et tantôt omet des formules comme "peut-être», «le plus souvent», "fait partie de», tantôt en ajoute. Dans certains cas, le sens en est affecté. Au milieu du paragraphe 12 du premier livre de La généalogie de la morale, le texte russe ajoute inopinément et sans raison apparente la formule «la race humaine empire» (p. 55). Ailleurs Preobrajenski parle de «nature humaine» (p. 66) ou enlève les guillemets autour de la formule "être humain». Ces libertés avec le texte ont une certaine importance, dans la mesure où le texte de Preobrajenski constitue le support essentiel de travail des premiers commentateurs qui le suivent, et citeront parfois ses formules comme étant de Nietzsche lui-même (c'est en particulier le cas d'Astafiev, citant la phrase «le sens et la valeur de la masse de la société ne sont pas renfermés en elle-même» [p. 62]).

En dehors du thème religieux, si l'on peut distinguer une tendance idéologique fondamentale dans l'article, c'est celle d'une atténuation du caractère nécessaire et systématique des logiques pulsionnelles, au profit d'un certain volontarisme. C'est peut-être aussi ce qui vaut à Aurore d'être privilégié par rapport aux textes postérieurs. Preobrajenski insiste sur l'opposition entre initiative individuelle et autorité morale, ainsi que sur des notions comme « raison» ou «jugement». Ainsi dans le paragraphe 338 du Gai savoir, Nietzsche parle d'une "nécessité personnelle» liée à «toute l'économie de [l']âme» (Preobrajenski ajoute une périphrase qui modalise la proposition: "sans même parler des cas où la souffrance s'avère être une nécessité personnelle» [p. 46]). À la page suivante, en résumant le paragraphe 30 de Par-delà bien et mal, Preobrajenski qualifie le « dédoublement de la souffrance» occasionné par la compassion de "superflu et inutile». Or en se reportant au texte nietzschéen, il apparaît que la question n'est pas tant pratique (savoir quelle conduite adopter, en déterminant si la pitié est souhaitable en tant qu'accroissement de la souffrance du monde, sans compensation positive) que «théorique»: une vision synthétique et 
englobante de tous les malheurs du monde conduirait-elle - nécessairement - ou non - à un tel dédoublement - comme le pense Schopenhauer :

Si l'on rassemblait tout le malheur du monde, qui pourrait se risquer à prononcer que de toute nécessité sa vue conduira par séduction et contraindra justement à la pitié, et donc au redoublement du malheur ${ }^{21}$ ?

Troisième exemple encore plus manifeste: dans le paragraphe $21 \mathrm{du}$ Gai savoir, Preobrajenski supprime la phrase selon laquelle les pulsions

[...] gouvernent avec une violence et une convoitise excessives et ne veulent absolument pas que la raison les contrebalance au moyen des autres pulsions ${ }^{22}$.

C'est surtout sur la question de l'instauration de nouvelles fins dernières qu'un décalage est sensible, par exemple lorsque Preobrajenski écrit:

Désormais, jusqu'à la construction de nouveaux idéaux de vie et d'action, la conduite d'un individu ne peut se soumettre qu'à sa propre raison, être gouvernée que par son propre choix, son goût et ses dons. (p. 58)

Les traits individualistes, voire l'aspect «anarchiste» du désordre des volontés particulières, sont ainsi systématiquement soulignés, au détriment de la logique propre, quoiqu'encore trop rudimentairement connue selon Nietzsche, du processus de transformation, largement collectif, qui est à l'œuvre. À plusieurs reprises, des références à la «conscience» sont ajoutées, et des références au «sentiment» supprimées ${ }^{23}$. Le lien entre l'investigation scientifique et l'application pratique est parfois estompé: Nietzsche écrit, en effet, au paragraphe que la formule que l'on vient de citer est censée résumer :

Reconstruire les lois de la vie et de l'action, - pour cette tâche nos sciences physiologiques et médicales, notre théorie de la société et de la solitude ne sont pas encore assez sûres d'elles: pourtant on ne peut tirer que d'elles les premières pierres des idéaux nouveaux (sinon ces idéaux nouveaux euxmêmes). (Aurore, $\$ 453$ )

Preobrajenski paraît considérer l'ensemble du corpus qu'il mobilise comme porteur d'une doctrine nietzschéenne homogène. Cela l'amène en particulier lorsqu'il superpose un passage des Considérations inactuelles à un passage ultérieur à utiliser plus volontiers les éléments qui dans les premières exaltent la liberté et le développement de la personne, et à

21. F. Nietzsche, Par-delà bien et mal, Paris, Flammarion (GF), 2000 (trad. par P. Wotling), $\$ 30$.

22. F. Nietzsche, Le gai savoir, Paris, Flammarion (GF), 1997 (trad. par P. Wotling), \$21.

23. Par exemple p. 62-63 dans la retranscription de Par-delà bien et mal, $\$ 258$. 
donner l'avantage à l'aspect prescriptif sur l'aspect descriptif, y compris lorsqu'il a affaire à des textes de La généalogie de la morale dont le caractère programmatique est discutable. C'est sans compter que sur certains points abordés, l'opinion de Nietzsche a changé du tout au tout, ainsi dans son appréciation du criminel entre Humain trop humain et Le crépuscule des idoles: Lou-Andréas Salomé signale dans sa monographie consacrée à Nietzsche qu'apparaît une valorisation du criminel qui passe du statut de «résidu " attardé d'une civilisation passée à celui de «monstre», comme figure annonciatrice et préalable nécessaire du surhomme. On peut illustrer ce «volontarisme» inspiré des Inactuelles par l'emploi qui est fait d'un extrait du chapitre VI de la Troisième inactuelle. Preobrajenski traduit approximativement:

Nous répondons nous-mêmes devant nous-mêmes de notre vie - nous serons les véritables timoniers de cette vie et ne la laisserons pas s'assimiler à une contingence dénuée de sens. (p. 58) ${ }^{24}$

Ce qu'il commente de la façon suivante:

Nous serons nos propres expérimentations et nos propres créateurs. Tâche élevée: élaborer à partir de soi une individualité entière et achevée. (p. 58)

Mais ce que Preobrajenski prend moins en compte, c'est que même dans la Troisième inactuelle, si l'homme doit effectivement devenir lui-même, ce «lui-même» est une sorte de destin qu'il ignore, mais dont la direction n'est pas moins déterminée par tout ce qu'il est déjà, ou plutôt, par tout ce qu'il a aimé, par les relations dans lesquelles il s'est engagé:

Que la jeune âme se retourne vers sa vie antérieure et se demande: "Qu'as-tu vraiment aimé jusqu'à ce jour, quelles choses t'ont attirée, par quoi t'es-tu sentie dominée et tout à la fois comblée? Fais repasser sous tes yeux la série entière des objets vénérés et peut-être te livreront-ils, par leur nature et leur succession, une loi, la loi fondamentale de ton vrai moi ${ }^{25}$.

Si Preobrajenski est peut-être plus proche dans l'esprit des Considérations inactuelles que des autres textes de Nietzsche, il ne tient certes pas compte de cette conceptualisation en termes de «loi». De la même façon, le passage de la Quatrième inactuelle convoqué par Preobrajenski (p. 59-60) ne mentionne pas Wagner, ni le contexte assez particulier de l'argument.

24. Preobrajenski traduit «nous assumons» pour «Wir haben zu verantworten» et omet "folglich».

25. F. Nietzsche, Troisième considération inactuelle, in Euvres, Paris, Gallimard (Bibliothèque de la Pléiade), 2000 (trad. par H.-A. Baatsch), t. I, p. 580. 
Malgré ces approximations, dont seul un échantillon est rapporté ici, force est de souligner la qualité des connexions et rapprochements intertextuels opérés par Preobrajenski, en particulier lorsqu'on pense qu'il s'agit d'une première étude sur la question. Le plan de l'exposé suit une progression très cohérente. Preobrajenski évoque dès la première phrase la «maladie mentale» de Nietzsche, mais sans en tirer autre chose que la possibilité d'un état des lieux. On ne commence à s'intéresser à Nietzsche que depuis peu, en Russie comme en Allemagne, et Preobrajenski avance une série d'explications, qu'il résume ensuite ainsi:

Il n'y a, en un mot, presque pas un seul aspect de la vie sociale et culturelle où Nietzsche ne se tienne en contradiction violente avec les idéaux et les partis qui dominent, éveillant de toute part une résistance instinctive par cet appel intérieur, que l'on entend constamment dans tout le travail de sa pensée et dans toutes ses manières de voir. (p. 36)

C'est donc au premier chef l'aspect contestataire et anticonformiste de Nietzsche qui est souligné, et ce sont, cette fois, les adversaires de Nietzsche qui voient leur conduite renvoyée à des causes indépendantes de leur volonté. Le facteur religieux de sa réception n'est cité qu'à la fin de la liste, après la critique de la science et de la civilisation industrielle, et les opinions politiques de Nietzsche. Le premier élément mentionné est «la négation irrévocable des idéaux moraux contemporains » et les "discours paradoxaux» qui l'accompagnent, ce qui s'accorde avec le sujet et la méthode de l'étude. En conséquence Preobrajenski se fend de quelques remarques sur le second point avant d'en venir au premier. $\mathrm{Si}$ «beaucoup ne se sont pas résolus à ranger Nietzsche au nombre des philosophes dans le sens étroit et habituel» (alors que Preobrajenski le présente d'emblée comme «philosophe, poète et compositeur»), cela est dû à l'absence dans son œuvre de la moindre «recherche achevée» consacrée à un domaine donné de la connaissance philosophique. Cependant «il n'y a presque pas un seul problème philosophique sérieux que Nietzsche n'ait abordé dans ses œuvres» (p. 36). Preobrajenski propose donc de «le mettre au nombre des moralistes, dans le vieux sens de ce mot, comme Pascal, La Rochefoucauld, Leopardi ou Schopenhauer ${ }^{26}$. Enfin, le préambule s'attache plus particulièrement à la personne du penseur, en évoquant sa "conscience intellectuelle», et en conséquence sa méfiance vis-à-vis des systématiciens et son indépendance

26. Nietzsche lui-même a largement contribué à encourager ce type de lecture, dont on trouvera un exemple récent dans le livre de Robert Pippin, Nietzsche moraliste français, Paris, O. Jacob, 2006. 
par rapport à ce qu'il a lui-même affirmé par le passé. Preobrajenski s'attache ainsi à apporter des gages de la valeur des textes par-delà la «perspective fluctuante des humeurs » :

Les livres de Nietzsche - ce sont des époques de sa vie personnelle et des impressions qu'il a éprouvées, leur unité est l'unité de sa personne, l'unité de l'humeur qui les traverse, l'unité de ces tendances fondamentales, qu'a suivies sa pensée. (p. 37)

Ses contradictions sont donc imputables à la «mobilité vivante de sa pensée» et à son "travail, comme sous-terrain, qui éternellement creuse et se détruit soi-même», ce qui vaut à Nietzsche d'être élogieusement comparé à Alexandre Herzen et à sa "casse impitoyable de ses illusions et de ses attaches les plus chères». La formule employée à cette occasion, le «tourment de la sincérité», pourrait bien être à l'origine du titre de la réponse de Lopatine: "Une sincérité maladive». Preobrajenski convoque pour illustrer son propos le poème $62 \mathrm{du}$ Prélude du Gai savoir («Ecce Homo»), dans lequel Nietzsche se compare à une "flamme insatiable» (p. 38).

Preobrajenski présente ensuite l'objet de sa recherche, il s'agit de la "critique [...] de la morale sociale ou de la morale de l'altruisme». L'équivalence posée entre ces deux termes ne va pas tout à fait de soi, puisque le mouvement «populiste», dominant dans les années 1860 en Russie, dont le reflux coïncide entre autres avec la montée en puissance du nietzschéisme, égalait plutôt l'action sociale à un égoïsme rationnel bien compris ${ }^{27}$. On retrouve un schéma semblable, qui reflète une évolution générationnelle, au début du roman de Piotr Boborykine: Pereval (Le passage) ${ }^{28}$, lorsque le personnage de Lyjine s'adresse aux tombes de ses aînés: "J'ai cherché le chemin pendant vingt ans [...] je ne crois plus à ce en quoi vous avez cru", et d'évoquer ses désillusions au sujet de l'idéal de citoyenneté, du paysan russe (moujik) et de la commune rurale (obchtchina).

La deuxième partie de l'exposé pose la moralité comme "problème » et non comme un donné qu'il faudrait fonder, ce qui suppose un investissement personnel fort: la morale était le domaine de prédilection de Nietzsche, la "tâche de sa vie», "son affaire personnelle»

27. C'est par exemple le cas dans le roman de Tchernychevski: Que faire?

28. Il s'agit peut-être du premier exemple de vulgarisation des idées nietzschéennes. Boborykine présente néanmoins sous un jour peu reluisant son personnage nietzschéen (Kostritsyne), auquel il prête en particulier un antisémitisme très marqué. Ce dernier se réclame de Nietzsche, et évoque plus précisément l'opposition des deux morales dans La généalogie de la morale et surtout le Zarathoustra. 
(Le gai savoir, $\$ 345$ ). En pratique cela nécessite une «collecte de petits faits ", une enquête sur d'autres temps et d'autres lieux, là où les moralistes ne connaissent d'ordinaire que leur entourage le plus direct (Par-delà bien et mal, $\$ 186)$. Cela suppose surtout de «se tenir plus haut que et en dehors de toutes les évaluations morales", "par-delà bien et mal» (Le gai savoir, $\$ 380$ ). On note au passage que la formule "plus haut que et en dehors de toutes les évaluations morales» sera reprise par Astafiev, or Preobrajenski ne relève pas le caractère problématique de l'entreprise signalé par Nietzsche dans le paragraphe: «la question est de savoir si l'on peut réellement arriver là-haut».

La troisième partie commence par définir et distinguer «moralité» et «moralité au sens subjectif», en se référant aux termes allemands («Moral» et «Moralität»). La morale est un «système de normes qui déterminent l'activité de l'homme», comprenant des exigences dont le respect conditionne la valeur des actes et des sentiments. Or il existe plusieurs normes et idéaux, et leur contenu peut fortement différer, sans compter qu'à l'intérieur d'un même système moral, il y a parfois des collisions de normes. La question se pose alors de savoir comment déterminer leur dignité relative. Celle-ci est liée à la valeur des «fins supérieures» qui orientent ces systèmes. Tout le problème est alors que:

Le critère de cette valeur ne peut déjà plus revêtir un caractère moral; il doit absolument reposer quelque part en dehors de la moralité. (p. 41)

Preobrajenski cite Aurore ( $\$ 139$ ) à l'appui. De plus, le degré auquel une prescription est obligatoire découle de l'acception volontaire des fins de la moralité, qui doivent être «clairement conçues et en outre reconnues» or il n'existe pas pour l'instant de "fin universellement reconnue» (Aurore, $\$ 108$ ), plus grave: c'est justement au sujet des questions les plus importantes que nous sommes le moins éclairés (Aurore, $\$ 107$ ).

Dans «l'ordre moral contemporain» (quatrième point), malgré l'opposition de fins dont personne ne semble remarquer l'incompatibilité comme l'existence la plus longue ou la libération maximale vis-à-vis de l'animalité, le bonheur suprême de quelques-uns et le bonheur moyen de tous (Aurore, $\$ 106$ ), une vue générale domine malgré tout: sont considérés comme moraux les actes dénués de motifs égoïstes ou cupides, qui sont animés par la compassion et l'aspiration au bien commun, «la sympathie», «l'abnégation, le sacrifice de soi». Cet altruisme, que Preobrajenski qualifie de «religion de la compassion » et de "religion de l'humanité», dérive de la morale religieuse chrétienne de la charité mais surenchérit sur celle-ci, qui demeurait essentiellement préoccupée du salut personnel (Aurore, \$132). 
Dans un cinquième temps, Preobrajenski aborde le «côté psychologique» et la "valeur morale» de la compassion. La critique nietzschéenne de la compassion s'attaque avant tout à sa définition par Schopenhauer (dans l'essai Le fondement de la morale) que Preobrajenski reformule ainsi: «sensation de la souffrance de l'autre comme s'il s'agissait de la sienne propre», "élimination même partielle de cette distinction qui existe entre deux individus». Selon Nietzsche il s'agit d'une erreur psychologique: pareil phénomène est davantage inventé qu'observé. D’abord il n'y a jamais d'oubli complet de soi dans nos actes (Aurore, $\$ 133$ ), et toute "compassion» est composée de nombreux éléments, dont une part de plaisir, en tant que satisfaction d'un besoin propre. De plus l'expérience nie que la compassion s'accompagne d'une compréhension de la souffrance de l'autre (Aurore, $\$ 334 ;$ Le gai savoir, $\$ 338)$. Exiger la compassion, c’est augmenter plutôt que réduire la somme des souffrances (Par-delà bien et mal, \$30), argument qui semble davantage inspiré par la référence à la Métaphysique des mours de Kant ( «Doctrine de la vertu», $\$ 34)^{29}$ que mentionne Preobrajenski (p. 47), que par le texte cité. Considérer l'autre comme un autre moi-même entraîne une perte de l'objectivité liée au détachement (Aurore, \$137), la compassion affaiblit et s'avère en définitive nuisible (Aurore, $\$ 134$ ); au lieu d'aider et de tranquilliser autrui, elle alourdit son fardeau en lui faisant porter de surcroît notre propre assombrissement (Aurore, $\$ 144$ ), et engendre une hostilité visà-vis de sa joie (Aurore, $\$ 80$ ), ou du moins s'accompagne d'une incapacité à s'en réjouir avec lui (Humain trop humain, $\$ 321$ ). Preobrajenski insère ensuite un développement qui ne semble pas tiré d'un texte en particulier: la compassion n'est pas la seule forme de sentiment sympathique, mais elle a un ton mélancolique qui lui est propre. Elle ne devient centrale que dans des civilisations dégénérescentes et fatiguées qui craignent plus que tout la souffrance et considèrent comme suprêmement vertueux tout ce qui la soulage (l'Inde, l'aube du christianisme, la civilisation européenne contemporaine).

Les sixième et septième parties examinent les deux motifs de la morale de l'altruisme: l'utilité et la crainte de la souffrance. Dans chacun des exemples d'altruisme que sont le chercheur (et son objet de recherche), l'amoureuse (et son amoureux), le soldat (et sa patrie), la mère (et son enfant), les sujets considérés cèdent à des penchants, se divisent et sacrifient une

29. E. Kant, «Doctrine de la vertu», in Métaphysique des mours, Paris, A. Durand, 1855 (trad. par J. Barni), $\$ 34$, p. 134: «Si un autre souffre et que je me laisse (au moyen de l'imagination) gagner par sa douleur, que pourtant je ne puis soulager, nous sommes alors deux à en souffrir, quoique (dans la nature), le mal n'atteigne véritablement qu'une personne. Or ce ne peut être un devoir d'augmenter le mal dans le monde, et par conséquent de faire le bien par compassion». 
part d'eux-mêmes à l'autre (Humain trop humain, $\$ 57$; Par-delà bien et mal, \$220). L'évaluation sociale positive vient de l'utilité: toute morale crée une hiérarchie en fonction de besoins et de conditions de conservation de la société. Est moral celui qui accomplit bien son rôle de fonction du troupeau (Le gai savoir, $\$ 116$ ). Pourtant les notions de «bien» et de «mal» ne sont pas les expressions naturelles d'une certaine conformité à un but, le mal (c'est-à-dire la haine, la joie méchante, le pillage, la soif du pouvoir) est aussi utile que le bien, quoiqu'autrement, à la conservation (Le gai savoir, $\$ 116$ ), voire davantage (Le gai savoir, $\$ 1$ ) par son rôle moteur et fondateur.

Malgré tout l'on considère la compassion comme utile; un deuxième élément doit donc rentrer en jeu. On loue celui qui se nuit en étant vertueux, parce que l'on considère que l'on évite par son sacrifice un mal plus grand à l'ensemble de la société. Ce qu'on plaint dans la perte d'un tel homme, ce n'est pas sa perte même, c'est l'outil que perd la société. On loue donc et sa fonction d'outil et la déraison qui l'aveugle à son détriment sur son intérêt personnel (Le gai savoir, $\$ 21$ ). On appelle «bien» ce qui va dans le sens d'une élimination des individus et les assimile aux membres d'un corps, même si l'on discute de la nature de ce corps (Aurore, $\$ 32$ ). On se trouve ici au cœur de l'article de Preobrajenski, qui consacre plusieurs lignes à reformuler les idées dans ses propres termes:

Le second signe caractéristique des sentiments et des actes sociaux se trouve dans leur influence dépersonnalisante ${ }^{30}$, dans leur tendance à niveler toute personnalité au niveau moyen de la masse sociale, dans l'aplanissement des âpretés individuelles, qui auraient rendu une forte personnalité trop inquiétante pour ses voisins et pour la société. (p. 52)

L'assentiment moral est donc lié en dernier ressort à la peur qu'inspire l'individualité. Est jugé bon ce qui affermit la sécurité collective et le sentiment de cette sécurité (Aurore, $\$ 173,174$ ). Mais il s'agit là de l'évaluation d'une société (comme celle de l'Europe contemporaine) qui s'est déjà attendrie et amollie au terme d'un processus progressif, au point de trouver toute punition superflue et injuste et de chercher à supprimer la peur par tous les moyens: au contraire, dans une société plus ancienne où l'on devait faire face à des dangers extérieurs, ce sont la dureté et la sévérité qui étaient valorisés (Pardelà bien et mal, $\$ 201)$. Notons que Preobrajenski précise dans une note:

Ce n'est pas l'attitude indulgente ${ }^{31}$ envers le criminel que Nietzsche condamne ici, mais son motif présupposé et sa cause - l’affaiblissement du sentiment. (p. 54)

30. «Обезличивающем».

31. «Мягкое». 
Preobrajenski convoque une série de textes d'Aurore, d'Humain trop humain de La généalogie de la morale, du Crépuscule des idoles, afin de soutenir que Nietzsche «rejette inconditionnellement quelque punition du criminel que ce soit», en tant qu'elle corrompt et le juge et celui que l'on juge: et «ne reconnaît qu'un seul jugement: celui du criminel sur soi-même; en pratique il plaide une libération complète du criminel de quelque responsabilité que ce soit». On voit clairement ici que le texte de référence pour Preobrajenski est décidément Aurore, et qu'il lit La généalogie de la morale à cette aune.

Ces différents éléments sont résumés dans la huitième partie par la notion de «morale grégaire». Les conséquences de cette dernière, telles qu'on les ressent aujourd'hui, sont un affaiblissement de la volonté, des instincts, des passions, au détriment du somptueux et du luxueux. Sécurité est devenu synonyme de fadeur, les petites jouissances ont éliminé la grande joie. L'égalitarisme a condamné les grands hommes à la solitude (Par-delà bien et mal, \$268). La décadence et la dégénération engendrent le dégoût, mais ouvrent aussi la perspective d'une «tâche nouvelle» (Par-delà bien et mal, $\$ 203$ ), résumée par la formule que Preobrajenski cite à plusieurs reprises: «on n'a pas encore épuisé en l'homme ses plus grandes possibilités».

Cette tâche, "la construction de nouveaux idéaux de vie et d'action", une réévaluation des valeurs, de nouvelles tables de la loi, est examinée dans la neuvième partie, qui revient sur la notion de «fin» exposée dans la troisième partie en insistant à nouveau sur la nécessité d'une libre acception et imposition. Mais pour l'heure, nous sommes aux prises avec l'ombre des vieilles idoles mortes (Le gai savoir, $\$ 108$ ), en attendant que vienne l'homme de l'avenir qui nous libérera (La généalogie de la morale, II, \$24).

Le problème se pose alors (dixième partie) d'un interrègne moral (Aurore, $\$ 453$ ), d'où la profession de foi individualiste que voici:

Si l'humanité n'a pas encore de but, qui déterminerait le sens de son existence, quelques obligations que ce soit peuvent-elles être imposées à l'individu, d'abdiquer sa personne, d'y renoncer pour un but inconnu, de se sacrifier aux intérêts d'un mécanisme social impersonnel et de son mouvement progressif et aveugle? Quelques limitations que ce soit de sa liberté et de son aspiration au développement et au perfectionnement d'après ses propres lois peuventelles surgir pour l'individu? Peut-on exiger de lui une soumission intérieure à quelque loi ou autorité extérieure à lui ? C'est que la personne est le principe et la fin de l'humanité. (p. 57)

Preobrajenski oppose les "chaînes des préjugés moraux » à «l'expression achevée» de la personne et des forces qu'elle renferme. C'est ici qu'il est fait appel à la Troisième inactuelle, en insistant sur sa dimension individualiste 
et volontariste. Il semble donc que la période de transition morale oblige au moins provisoirement à reposer le problème à l'échelle de chaque individu : il s'agit de «devenir soi-même» et de trouver le contentement de soi pour ne pas se venger de son mécontentement sur les autres (Le gai savoir, $\$ 270$ ). Au fond cette oscillation entre discipline personnelle et projet civilisationnel se trouve également chez Nietzsche lui-même ${ }^{32}$. Dans une société qui estime l'individualité, apparaissent des hauts faits dont l'éclat est durable (Aurore, $\$ 529$ ), parce que l'on s'imagine que nos pulsions sont mauvaises, l'aristocratisme de l'âme est rare (Le gai savoir, \$ 294). Toute morale exclusive détruit de bonnes forces, celles de ceux qui refusent la société et vivent comme ils l'entendent (Aurore, $\$ 164$; Preobrajenski fait référence en note à la mention à Dostoïevski du Crépuscule des idoles: «Incursions d'un inactuel» [\$ 45$]^{33}$ ), il faut donc laisser subsister les antipodes du globe moral (Le gai savoir, $\$ 289$ ). Certes, en abaissant le prix des «actions morales», on les rendra plus rares, mais on rétablira ainsi l'équilibre en rendant le courage d'accomplir les actions «égoïstes» sans mauvaise conscience: en cessant de se considérer comme mauvais l'homme cessera de l'être (Aurore, $\$ 148$, 202; une note apporte l'exemple des pulsions sexuelles: Aurore, $\$ 76$ ). Mais cet interrègne ne sera pas un «âge d'or» de «bonté surhumaine», l'homme semblera peutêtre même plus méchant. Cependant on y reconnaîtra que «la passion vaut mieux que le scepticisme", "qu'il vaut mieux être honnête et sincère, même dans le bien, que de s'incliner devant la moralité traditionnelle ", que le plus important est d'être libre, ce qui nécessite de se rendre libre. Preobrajenski utilise ici un extrait du dernier chapitre de la Quatrième inactuelle, ce qui révèle à nouveau une certaine continuité problématique entre les Inactuelles et les œuvres ultérieures, mais fait abstraction du contexte spécifique du texte.

La onzième partie examine à travers les notions de surhomme et d'aristocratie s'il n'existe pas pourtant de tâche qui dépasse l'individualité. Aucun but n'est déterminé à l'avance. Ici une note faisant référence au Gai savoir $(\$ 357)$ permet de mesurer l'écart entre Preobrajenski et le mot de la rédaction:

[Nietzsche] remarque de façon tout à fait juste que ce n'est qu'avec la décadence des croyances religieuses qu'a pu s'élever sous une forme véritable, avec tout son poids oppressant, la question de la valeur de la vie. (p. 6o)

32. Voir F. Nietzsche, Troisième considération inactuelle, chap. I, p. 579: «Mais même si l'avenir ne nous laissait aucun espoir, la singularité de notre existence en ce moment précis est ce qui nous encourage le plus fortement à vivre selon notre propre loi et selon notre propre mesure».

33. «Nietzsche, signale, entre autres, les types, tirés de La Maison des morts de Dostoïevski, qu'il considère comme le plus profond des psychologues qu'il connaisse» (p. 59). 
Mais la formulation précise de la question, que Nietzsche attribue à Schopenhauer, est «l'existence a-t-elle seulement un sens?», et à nouveau Preobrajenski paraît se garder de citer textuellement la formule «le déclin de la croyance au Dieu chrétien». En l'absence d'un but pré-existant,

[...] ce n'est que par sa volonté que l'homme investit un sens dans sa vie il crée lui-même sa signification et son sens et, pour ainsi dire, la justifie $a$ posteriori. (p. 6o)

Or il n'y a pas de meilleur but dans la vie que de mourir pour quelque chose de grand (Seconde inactuelle, chap. IX). Les chaînes des erreurs morales, religieuses et métaphysiques, sont des séquelles d'animalité en l'homme, dont il doit achever de se défaire (Le voyageur et son ombre, $\$ 350$; Preobrajenski recommande de se reporter à La généalogie de la morale, II et III au sujet de ces "chaînes"). C’est ce dépassement de soi et le sens qu'il confère à l'existence terrestre qu'illustre alors la formule «Le surhomme est le sens de la terre» (Ainsi parlait Zarathoustra, "Prologue de Zarathoustra», $\$ 3$ ). Cependant les hommes ne sont pas égaux: il y a un ordre hiérarchique et des différences de rang (Le crépuscule des idoles, «Incursions d'un inactuel», $\$ 48$ ), d'où la nécessité d'une pluralité de morales (Par-delà bien et mal, $\$ 228$ ). La pire injustice serait que la vie la moins développée se donne comme fin et mesure des choses et mine ce qu'il y a de plus élevé (Humain trop humain, Préface, $\$ 6$ ), certains ont plus droit que d'autres à l'existence, distinction qui doit être maintenue par le pathos de la distance (La généalogie de la morale, III, \$14). Ce que Preobrajenski résume ainsi: «ce serait faire preuve d'une prodigalité insensée que de faire de ce qui est sain un instrument de ce qui est malade ou du génie un instrument de la masse ", formule qu'Astafiev citera en l'attribuant à Nietzsche. Or l'immense majorité de l'humanité n'a ni pensée ni volonté propre, son indifférence en fait un obstacle, ce n'est pas en elle qu'est le sens de l'humanité (Preobrajenski cite la Seconde inactuelle, chap. IX, à l'appui). Elle est comme le chêne dont se servent les plantes parasites de l'île de Java (Par-delà bien et mal, \$258). L’humanité ne peut donc s'élever que via une société aristocratique: il faut creuser l'écart entre hommes pour élargir l'âme elle-même (Par-delà bien et mal, \$257).

L'idéal de la création s'oppose alors à tout eudémonisme (douzième partie). Cet idéal vaut que l'on se sacrifie soi-même et que l'on sacrifie les autres pour lui (Aurore, $\$ 146$ ). Si on lui montre un sens à la souffrance, l'homme se réconcilie avec elle (La généalogie de la morale, III, $\$ 28 ; L e$ crépuscule des idoles, "Maximes et flèches", \$12). Le plaisir et la souffrance ne sont donc pas la mesure dernière des choses, la souffrance ne doit pas être éliminée, elle est condition de la grandeur (Par-delà bien et mal, \$255), de 
même que le danger vaut mieux que la sécurité (Par-delà bien et mal, $\$ 43$; Le crépuscule des idoles, $\$ 44)$. L'homme unit en lui créateur et créature, la créature doit souffrir, car elle sert de matière qu'il faut mettre en forme (Par-delà bien et mal, \$225).

C'est en ce sens qu'il faut entendre (treizième partie) le «nouveau commandement» de la fable du diamant et du charbon (Ainsi parlait Zarathoustra, «Des vieilles et des nouvelles tables», $\$ 29$ ), et sa morale: «faites-vous durs».

Quatorzième point: à la question posée au début dans sa troisième partie (en quoi consiste le critère extra-moral qui détermine la valeur des idéaux moraux?), Preobrajenski peut à présent répondre:

C'est la vie, son énergie et son épanouissement, qui servent de critère de la morale, et non l'inverse. (p. 66)

C'est de ce point de vue qu'il faut considérer l'éloge par Nietzsche de César Borgia et de la Renaissance (Le crépuscule des idoles, "Incursions d'un inactuel», \$37). Preobrajenski conclut l'article en insistant sur les liens entre sa problématique et la problématique d'ensemble de l'œuvre, qu'il résume par la formule «critique et approfondissement du pessimisme» (sans doute inspirée de Par-delà bien et mal, \$56):

Nous avons vu comment se fait le passage de la question même de la valeur de la morale à la question de la valeur de la vie. (p. 69)

On le voit, le premier article russe consacré à Nietzsche laisse essentiellement la parole à Nietzsche lui-même, ce qui est une façon non équivoque de l'approuver, et ne comporte pas une seule critique des vues rapportées. De fait Preobrajenski était à ce point perçu par ses contemporains comme un nietzschéen qu'il a servi de prototype au personnage de Kostritsyne dans le roman de Boborykine, Pereval (Le passage) (1893) et qu'à sa mort en 1900, plusieurs articles dans Questions de philosophie et de psychologie ont évoqué cet aspect de son personnage, dont celui de Boborykine: «Sur le nietzschéisme». Il est notable aussi que cet article soit le fait d'un philosophe de formation, qui a enseigné la philosophie, traduit et publié Leibniz et Spinoza, et travaillé aussi bien sur Spencer que sur Schopenhauer. À la lumière des quelques passages qui sont dus à Preobrajenski lui-même dans le corps de l'exposé, on peut avancer que l'idée centrale du texte est l'affirmation d'un droit à l'auto-détermination en matière morale, qui, au-delà d'une défense des spécificités individuelles contre la société et sa médiocrité, prend la forme d'une réaction aristocratique à l'idéal «social» des décennies précédentes. 


\section{Lopatine}

Trois articles répondent à Preobrajenski dans le numéro suivant de la revue Questions de philosophie et de psychologie $\left(\mathrm{n}^{\circ} 16\right)^{34}$.

Le premier est dû à Lev Mikhaïlovitch Lopatine ${ }^{35}$. Quatre à cinq fois plus court que les deux autres, il est centré autour d'un unique argument. Nietzsche est perçu comme le dernier représentant d'un courant tricentenaire: «un scepticisme téméraire, qui ne recule devant rien» et "constitue la tendance la plus caractéristique de la philosophie moderne ». Son principal mérite à ce titre est d'être un sceptique conséquent. En effet, «une multitude immense d'hommes contemporains» refuse «l'existence de l'espace et du temps; toute dépendance entre les choses, et entre leurs phénomènes ", considérés comme des illusions d'un appareil cérébral luimême inexistant, notre moi, et le monde, à titre de «songe subjectif sans fondement ». On considère comme naïve illusion métaphysique l'idée que la pensée et la volonté ont une influence sur l'action, chez nous comme chez notre prochain. Cet «ensemble bariolé de négations étonnantes et fantasques » est considéré comme "sagesse humaine suprême » et baptisé «du nom inspiré de philosophie critique». Mais, remarque-t-il, «nous changeons vite de ton quand l'affaire touche aux questions morales». Même si l'on interprète diversement les fondements de la morale, son but suprême, on ne se décide pas pour autant à porter atteinte au contenu même des prescriptions "généralement reçues", le résultat de cet apparent consensus, c'est que «l'idéal humaniste chrétien se dresse aujourd'hui peut-être plus haut que jamais». Si ce fait se passe de justification pratique, sur le plan théorique il s'agit d'une inconséquence manifeste, une «double comptabilité», et c'est en cela (et en cela seulement) que l'on peut donner raison à Nietzsche. Car selon Lopatine:

Si nous pensons la réalité d'une certaine manière, nous nous rapportons inévitablement à elle de la même façon: une négation théorique ne peut pas ne pas se refléter sur les fins de notre activité. (p. 73)

34. Les références précises de la publication sont les suivantes: Вопросы философии и психологии, № 16, 1893: «Больная искренность » (р. 109-114), «Нравственные идеалы нашего времени » (р. 129-154), «Генезис нравственного идеала декадента ( (р. 56-75).

35. Лев Михайлович Лопатин (1855-1920), professeur à la faculté d'histoire et de philologie de l'université de Moscou, est l'un des premiers représentants du personnalisme russe. Il a tenté à travers ses travaux de remédier au «dogmatisme » de la philosophie de la fin du $\mathrm{XIX}^{\mathrm{e}}$ siècle, par un renouveau de l'ontologie, qui la reconduise aux notions fondamentales les plus générales. Il qualifiait sa philosophie de "spiritualisme moniste» ou «concret» dans son ouvrage Положительные задачи философии (Les problèmes positifs de la philosophie). 
Pas de devoir sans «moi» ni «liberté». Si toutes les distinctions comme le bien et le mal ne sont que des inventions, dont le processus historique nous montre l'origine dans les impulsions animales les plus basses, si les obligations ne sont produites par la société que pour changer les personnes en instruments aveugles, quelles obligations peuvent encore exister envers le prochain?

Faisons taire notre conscience et nous serons libres. Voilà ce que nous prêche

Nietzsche dans ses aphorismes sincères. (p. 73)

C'est là ce que Lopatine appelle la "sincérité maladive de la négation nietzschéenne » : Nietzsche serait le seul à tirer les conséquences pratiques des théories sceptiques. Il est donc avant tout l'exemple édifiant de l'impasse vers laquelle tend tout scepticisme, et «la conception du monde du mécanisme naturaliste». L'article s'achève sur un appel à la tâche "réellement critique» de la philosophie contemporaine: il s'agit de se rapporter aux tâches théoriques également « de façon plus vive et plus sérieuse». Nietzsche a contribué à la position des problèmes, et c'est là l'explication réelle du «charme» qu'il exerce sur nous.

Néanmoins dès qu'il évoque l'aspect "positif» de la philosophie de Nietzsche, Lopatine souligne à l'inverse son inconséquence: «le caractère déchaîné, peu sérieux, et pourrait-on dire hystérique de ses conclusions finales» montre qu'aucune morale n'est possible de son point de vue. Le caractère achevé, entier et conséquent de Nietzsche que soulignait Preobrajenski n'est donc qu'apparent, et ses contradictions sautent rapidement aux yeux. D'abord Nietzsche commence par affirmer qu'il n'y a pas de fin universellement reconnue avant de prôner lui-même une fin universelle (la puissance et la magnificence la plus grande du type humain), et ce au prix de la condamnation à l'esclavage permanent de la majorité écrasante des hommes, justifiée à leurs yeux par une éducation. En second lieu l'homme est à la fois conçu comme «créateur libre» et comme «jouet automatique d'inclinations déchaînées » - ce dernier point est d'ailleurs le seul véritable élément chez Lopatine qui ne se trouve pas ou du moins qui ne soit pas mis en avant dans le texte de Preobrajenski. Nietzsche n'est donc ni logique ni rigoureux, son argumentation, si tant est qu'il y en ait une, est très faible, ce qui est en bonne partie dû à la forme aphoristique. Ses procédés démonstratifs se limitent à des rapprochements et à l'invocation de faits particuliers. En un mot, malgré sa "profonde originalité» et son «courage», on n'a affaire qu'à un «lyrisme subjectif de la pensée», brillant et fantasque.

Pointant par sa sincérité les conséquences pratiques inévitables et néfastes du scepticisme théorique, Nietzsche possède donc une utilité toute négative. On remarque au demeurant que, bien qu'elle relève des traits 
du nietzschéisme considérés comme scandaleux, la critique de Lopatine est entièrement ordonnée au principe logique de la conséquence des arguments. Quoi qu'il en soit, Lopatine témoigne de l'impact de l'article auquel il répond, qui selon lui «a particulièrement attiré l'attention des lecteurs et a produit une assez forte impression».

\section{Grot}

La seconde réponse, intitulée "Les idéaux moraux de notre temps (Friedrich Nietzsche et Lev Tolstoï)», est signée Nikolaï Yakovlevitch Grot ${ }^{36}$. Grot construit une antinomie entre les deux penseurs qu'il examine, qui témoignent pour lui d'une situation de crise morale («un grand drame de l'âme») en réaction à laquelle ces deux penseurs constituent deux réponses également « unilatérales» et excessives, quoique de façon inverse. Ce qui entre ainsi en crise, c'est le compromis, établi trois siècles auparavant, entre christianisme et paganisme, et cette crise est elle-même la troisième du genre, la première datant de la fin de l'Antiquité et ayant marqué l'avènement du christianisme, la deuxième du XVI ${ }^{\mathrm{e}}$ siècle, avec l'effondrement du monde chrétien médiéval et le début d'un compromis entre valeurs chrétiennes et païennes. Sa spécificité est qu'elle advient dans un monde qui tourne beaucoup plus rapidement, ce qui est lié aux changements sans précédents dont le XIX ${ }^{\mathrm{e}}$ siècle a été témoin, en particulier en ce qui concerne les moyens de communication et de transport. La première partie de l'article est consacrée à une description de cet état de fait inédit: toutes ces inventions constituent un «nouveau système nerveux» pour l'humanité, qui devient grâce à elle un "organisme». L'histoire, comme science, constitue sa «mémoire organisée». Ces changements ont selon Grot une dimension morale essentielle, tous les événements, toutes les conceptions, sont bientôt connus, les fautes éclatent au grand jour, on gagne en transparence, et la difficulté croissante du mensonge va de pair avec une augmentation de la responsabilité personnelle.

36. Николай Яковлевич Грот (1852-1899), président de la Société moscovite de psychologie, et l'un des premiers instigateurs de la revue Questions de philosophie et de psychologie. Son activité philosophique se divise en deux périodes. La première (1879-1884) est marquée par le positivisme, l'utilitarisme, des recherches en logique, qui récusent toute forme de métaphysique. La seconde, baptisée "monodualisme», qui s'appuie sur la philosophie antique et renaissante, privilégie à l'inverse la méthode subjective, la métaphysique, et s'appuie sur le sentiment de la vie universelle. Les notions de «force» et de la «volonté» deviennent alors centrales, en particulier sous l'influence de Schopenhauer, puis du chimiste Wilhelm Ostwald, mais la synthèse opérée par Grot rassemble en réalité des auteurs d'horizons très différents. 
Dans ce contexte le succès des nouveaux enseignements sur la vie tient à la «soif de nouveaux idéaux» qui travaille les hommes, «tous plus ou moins malades de scepticisme, tous pleins de dégoût pour l'ordre moral existant». Car plus ces progrès vont croissant, plus éclatent au grand jour l'absurdité et l'inconséquence du compromis entre idéaux païens et chrétiens, deux principes de vie opposés et incompatibles. Il se pose donc un dilemme, beaucoup plus difficile à résoudre qu'il ne pourrait y paraitre. Mais il n'est pas question d'un choix de chacun fondé sur des préférences personnelles: à mesure que le lien entre les hommes devient plus fort, l'unité de leur conception du monde se fait plus nécessaire.

Or les acquisitions de la science ont surgi sur le sol du paganisme. Mais le progrès moral qu'elles ont amené en qualité de «moteur de la conscience de soi» est tel que l'on n'est ni en droit ni en état de rejeter ce fondement. À l'inverse, la conception morale du monde du christianisme surpasse à tel point celle du paganisme, elle a si profondément pénétré la vie, qu'il serait «suicidaire» d'y renoncer. Trois voies s'offrent alors: soit l'on opte pour le christianisme, soit l'on opte pour le paganisme, soit l'on tente une réconciliation par de nouvelles voies et sur une nouvelle base. Mais les propositions qui vont en ce dernier sens sont restées jusqu'alors faibles et peu convaincantes.

C'est dans ce contexte que Grot lit Nietzsche comme le défenseur d'une conception du monde purement païenne, en le comprenant comme l'antithèse exacte de Tolstoï. En réalité, il faut savoir que son article vise à réhabiliter Tolstoï auprès des autorités religieuses et à lui éviter l'excommunication - ce fut d'ailleurs un échec. Grot construit son étude sur une lecture qui semble souvent linéaire de larges passages de La généalogie de la morale, en effet on peut suivre à plusieurs reprises l'apparition parallèle des notions dans son texte et dans celui de Nietzsche. Sa lecture de Nietzsche est en bonne partie conditionnée par la volonté d'y trouver toujours à nouveau le contradicteur de Tolstoï, auquel il accorde une nette préférence, malgré les critiques qu'il lui adresse à la fin de son article pour s'en démarquer.

Grot commence par repérer les points communs des deux doctrines, pour mieux se concentrer ensuite sur leurs dissensions. Chez Nietzsche comme chez Tolstoï, il identifie une double protestation, contre la conception morale du monde contemporain, et contre l'organisation séculaire traditionnelle de la société chrétienne, tous deux dénonçant ses vices cachés et son hypocrisie. En découle chez l'un comme chez l'autre une lutte contre l'Église et l'État, coupables supposés de cet état de fait. Tous deux partagent également un individualisme, une aspiration au triomphe de la raison, à libérer les personnes des conventions, à élever leur conscience de soi, bref à créer des personnes nouvelles, plus libres, indépendantes, comme base d'une nouvelle société. La cible de leurs attaques respectives est pourtant très différente. 
Tout le mal repose pour Nietzsche dans les fers et chaînes intérieurs imposés par la conception du monde morale et religieuse chrétienne. Le christianisme s'est déjà désagrégé en bonne partie comme religion, il doit à présent périr également comme morale. Il s'agit de libérer l'homme de la mauvaise conscience, de la responsabilité morale, de laisser dominer les passions pour que s'épanouisse la personne, jusqu'à ce que les hommes supérieurs atteignent le surhomme, comme nouvelle espèce de l'animalhomme, tandis que les masses lui serviront d'instrument passif.

Il est tout à fait évident [...] qu'il rêve d'un retour aux sources et aux principes de la culture païenne. (p. 81)

D'où son admiration pour l'Antiquité et la Renaissance. Grot nuance toutefois en se faisant l'avocat des intentions de Nietzsche:

Bien sûr, ce ne sont pas les vices et les crimes, la débauche et les violences, que Nietzsche admire mais l'épanouissement parallèle de la génialité et de la créativité $[\ldots]$ cependant il considère tous les phénomènes négatifs indiqués comme le revers inévitable et insurmontable de la médaille. (p. 81)

Cela signifie aussi que sa doctrine ne peut être perçue que comme un égarement étrange, et en effet, Grot compare Nietzsche à un miroir déformant, qui reflète toutes les choses en leur conférant une apparence paradoxale qui ne leur est pas fidèle. Si ce n'est pas une perversion de nature morale qui pousse Nietzsche à écrire ce qu'il écrit, il n'est pas responsable moralement. Il ne reste alors qu'à le déclarer «le plus fou de tous les hommes de l'hôpital universel et de la maison de fous qu'il a imaginés", ce qui pousse Grot à lui attribuer le portrait de l'homme du ressentiment tel qu'il l'a lui-même dépeint ${ }^{37}$, et à ce titre Nietzsche est digne de "pitié», en tant qu'il a «vécu une des tragédies les plus pénibles - la tragédie morale de l'incroyance et de la négation, et a eu le courage de confesser sincèrement devant l'humanité tout ce qu'il a médité et enduré». Cette dissymétrie de sa vision des choses, son caractère paradoxal, se retrouvent alors dans la formule qui résume l'enseignement de Nietzsche:

Plus il y a de mal, plus il y a de bien. (p. 81)

Le mal remplissant la fonction de l'indispensable fond noir du "tableau » de la libération de la personne.

37. F. Nietzsche, La généalogie de la morale, III, \$14, p. 219; la mention de l'hôpital se trouve d'ailleurs deux pages plus tôt. 
Pour Tolstoï au contraire, le mal n'est pas à l'intérieur, mais «dans les chaînes extérieures de l'organisation sociale", il est lié au caractère insuffisant du renoncement au mode de vie païen. Seul le christianisme constitue un gage de développement spirituel véritable de la personne. Cependant comme Nietzsche, Tolstoï pense qu' 'il n'y a pas lieu de chercher une fin et un sens à la vie dans une tâche transcendante de rachat de l'âme du péché, mais avant tout dans la meilleure organisation de la vie spirituelle d'ici-bas" (p. 82). Au lieu de détruire la conscience, il faut la développer, et l'inverse vaut de l'égoïsme. Il ne s'agit pas de perfectionner le type humain, mais de développer les forces cachées de l'homme lui-même, qui est déjà un «sur-animal». Il faut revenir dans la foule de ses semblables et niveler les inégalités sociales au lieu de les renforcer. Grot accorde que c'est là l'idéal d'un animal domestique pacifique, qu'il distingue cependant de l'animal de troupeau. La formule tolstoïenne, qui est au contraire un signe positif de santé, prend le contre-pied de celle de Nietzsche:

Moins il y a de mal, plus il y a de bien. (p. 82)

Dans les faits ces conceptions les amènent à des jugements opposés sur le compte de l'ascétisme. Grot s'appuie ici sur la troisième partie de $L a$ généalogie de la morale, en accusant Nietzsche de "discréditer injustement le sens moral» de l'ascétisme, en réduisant l'abstinence à une question d'hygiène ou de diététique. Nietzsche chercherait ainsi à substituer à l'idée d'un perfectionnement de soi spirituel et moral la transformation de l'homme en une nouvelle espèce d'animal - ceci alors même que Nietzsche parle au milieu du passage cité de "suprême spiritualité» ${ }^{38}$. Grot fait allusion aux passages de Nietzsche sur Luther, le Parsifal de Wagner et Feuerbach (chapitres 2 et 3), puis sur Socrate et le mariage et «l'ascétisme gai» (chapitres 7 et 8 ). Ce qu'il considère comme condamnable, même s'il relève comme un point positif l'analyse de l'ascétisme comme affirmation de la volonté de vie (contre Schopenhauer), c'est de ne voir en lui qu'un moyen, un idéal «faute de mieux ${ }^{39}$ et de le considérer comme une "condition" (et non pas comme une vertu, si l'on se reporte au texte de Nietzsche). Mais, ce faisant, Nietzsche ne voit dans l'ascétisme qu'un médicament contre la dégénération, et exclut son emploi préventif - il rate ainsi la distinction entre deux altruismes, l'altruisme affaibli, qu'il a raison de critiquer, et une compassion virile. Surtout, considérer l'ascétisme comme une condition de vie quelconque (donc la réponse à un certain

38. F. Nietzsche, La généalogie de la morale, III, \$9, p. 202.

39. L'expression est en français dans le texte. 
besoin) suppose une assimilation de l'homme à l'animal, et Grot mentionne quelques occurrences qui associent avec une " délectation étrange» les termes Thier et $\mathrm{Mensch}^{40}$. Bien que Tolstoï soit également étranger à l'idéal monastique médiéval, il prêche à l'inverse l'abstinence, en même temps qu'il condamne les nourritures carnées, le vin, le tabac et autres narcotiques, dans son entreprise de simplification de la vie, et surtout il distingue radicalement l'homme et l'animal.

En ce sens Tolstoï et Nietzsche s'opposent comme «le porteur des idéaux de la spontanéité ${ }^{41}$ de l'Europe orientale» au «représentant de l'affectation de l'Europe occidentale». Grot poursuit plus loin dans la même veine:

Malgré toute la haine et le dégoût de Nietzsche pour la civilisation contemporaine industrielle et bourgeoise, l'écho parait malgré tout dans son enseignement du bruit continuel et du fracas des machines d'une immense usine d'Europe occidentale ou d'Amérique. (p. 87)

Nietzsche se voit ainsi attribuer une sorte de progressisme scientiste, le surhomme étant perçu comme le produit d'une «élaboration mécanico-chimico-physico-anatomo-physiologique» et décrit comme une "créature-volante et ailée », peut-être en relation avec l'expression " animal divinisé, ayant appris à voler de ses propres ailes, qui plane au-dessus de la vie plutôt qu'il ne s'y repose», qui dans La généalogie de la morale ${ }^{42}$ n'est pas une description du surhomme, mais de l'image que «les philosophes» associent à l'ascétisme comme ascétisme gai. Et Grot d'entendre au contraire chez Tolstoï :

[...] la résonance des espaces de steppe silencieux, étendus, peu travaillés de notre patrie, des champs de terre noire infinis et mélancoliques, de l'isolement tranquille et recueilli de la campagne, dans lequel on ressent vivement le «pouvoir de la terre» et «la liberté d’un esprit sain et puissant dans son isolement». (p. 88)

La tension entre cette image d'un Nietzsche hyper-moderniste et son désir souligné de revenir à l'Antiquité païenne ne paraît pas troubler outre mesure Grot, quand bien même Nietzsche la souligne quelques pages plus loin:

40. Il s’agit de passages situés dans F. Nietzsche, La généalogie de la morale, I, \$1, p. 66; III, $\$ 15$, p. $222 ;$ III, $\$ 28$, p. 270.

41. «Непосредственность» : littéralement «l'immédiateté». Nietzsche est également associé dans le texte à un mot que l'on peut traduire «spontanéité»: «стихийность», mais c'est de la spontanéité des éléments et des catastrophes naturels qu'il s'agit.

42. F. Nietzsche, La généalogie de la morale, III, \$ 8, p. 197. 
Selon l'étalon des Grecs de l'Antiquité, tout notre être moderne [...] offre l'aspect de l'hybris [...]. Hybris est aujourd'hui toute notre position à l'égard de la nature, notre façon d'exercer des violences sur la nature à l'aide des machines et de l'inventivité si dénuée de scrupules des techniciens et des ingénieurs [... $]^{43}$.

Autrement dit la lecture de Nietzsche est entièrement ordonnée à la fin pratique d'apporter un remède immédiat aux problèmes identifiés en début d'article. Grot accentue l'aspect binaire de La généalogie de la morale, en ne remarquant pas que l'analyse développée par Nietzsche ne se limite peut-être pas à une prise de position et à un enseignement moral exigeant une application immédiate. Il lit donc Nietzsche en y cherchant l'équivalent de ce qu'il trouve chez Tolstoï: un sermon prescriptif immédiatement applicable et convertible en conduites concrètes. C'est ainsi que le mérite de Tolstoï tient à ce qu'en reprochant à un homme de boire et de fumer, en lui faisant honte, il produit l'effet très concret de l'amener à arrêter de boire et de fumer. On peut se demander s'il est possible de faire une lecture similaire de Nietzsche, orientée par des exigences de résultats tangibles. Mais peut-être est-ce aussi l'intérêt d'une critique de ce genre que de montrer quelles questions très simples les lecteurs contemporains pouvaient poser aux textes de Nietzsche et quelles réponses ils en déduisaient: une personne avide de conseils pratiques pourrait comprendre La généalogie de la morale comme l'injonction d'étouffer tout remords de conscience et tout amour du prochain.

Nietzsche est considéré comme un esthète, dont le rationalisme est ordonné à la "perfection extérieure de la forme», au «charme de la beauté» - comme le suggérait la métaphore du «tableau» -, tandis que Tolstoï, qui soumet la raison à la morale, et pour qui l'homme est tout entier raison pratique, commet l'erreur inverse de rejeter en bloc toutes les formes extérieures - l'art, la science, la religion dogmatique, la politique (p. 92-93) -, alors que celles-ci sont les incarnations nécessaires des idées divines. En lieu et place de l'exclusivisme qui amène chacun des deux penseurs à ne retenir qu'une des trois idées divines, le beau (Nietzsche), le bien (Tolstoï) et la vérité, il faut rétablir leur égalité de droits.

Les besoins de l'argumentation amènent à plusieurs reprises Grot à qualifier Nietzsche de "matérialiste» pour l'opposer à «l'idéaliste» Tolstoï; pour Nietzsche il n'y aurait rien dans le monde «à part des substances et leurs combinaisons", "l'homme est une machine», "toutes les actions de l'homme sont les produits d'un mécanisme compliqué». On lit également par exemple:

43. F. Nietzsche, La généalogie de la morale, III, \$19, p. 203. 
Nietzsche est un anarchiste révolutionnaire et, comme tout révolutionnaire, un dogmatique du despotisme. (p. 84)

Affirmation bâtie en miroir sur celle qui suit pour filer l'effet de symétrie :

Tolstoï est l'ennemi le plus décidé de l'anarchie, de la révolution et du despotisme. (p. 84-85)

Nietzsche prônerait ainsi l'emploi d'une violence habile, tandis que Tolstoï refuse toute violence au nom du principe de non-résistance au mal.

On note que dès que Grot ne s'appuie plus sur un texte précis de Nietzsche, ou tente des généralisations catégoriques, ses affirmations acquièrent un caractère relativement hasardeux :

Nietzsche est un matérialiste ${ }^{44}$, un athée et un évolutionniste. (p. 86)

Trio de notions repris à deux reprises, associé à la négation de l'âme (à travers celle du sujet ${ }^{45}$ ) et à celle de Dieu ${ }^{46}$, et renvoyé comme à leur conséquence au "tout est permis» d'Ivan Karamazov dans le roman de Dostö̈evski $^{47}$ (p. 87). L'objectif de Nietzsche consisterait non pas à prendre le contre-pied de la culture contemporaine, mais à exacerber la «lutte pour l'existence», visiblement prise ici au sens d'un darwinisme vulgarisé. Tolstoï à l'inverse croit en Dieu, croit à la survie de l'âme, même s'il la présente parfois sous la forme de l'immortalité d'un esprit impersonnel, et affirme que toute velléité révolutionnaire et toute lutte disparaîtraient si l'homme était tout à fait libéré:

Toutes les forces supérieures de la personne n'apparaîtront que lorsqu'elle renoncera elle-même de son plein gré à toute puissance et force, à toute violence légitime. (p. 85)

Le point séminal du désaccord entre les deux penseurs se situe pour Grot dans leur conception de la nature humaine: l'homme de Nietzsche est le plus méchant des animaux, c'est pourquoi il en vient à penser qu' "après avoir dévoré une certaine quantité de ses prochains et sucé la sève de dizaines ou de centaines de ses semblables » il se surpassera et deviendra un surhomme. L'homme de Tolstoï est bon tant qu'on ne le rend pas méchant.

44. Nonobstant d'ailleurs ce qu'écrit Nietzsche à quelques pages des passages cités par Grot: «avec une telle conception, on peut rester, soit dit en passant, le plus strict adversaire de tout matérialisme» (F. Nietzsche, La généalogie de la morale, III, \$16, p. 227).

45. F. Nietzsche, La généalogie de la morale, I, \$13, p. 97-98.

46. Ibid., III, \$27, p. 267.

47. F. Dostoïevski, Les frères Karamazov, Paris, Actes Sud, 2002 (trad. par A. Markowicz), t. I, p. 130 sq. 
Tout le tort de Tolstoï est selon Grot dans l'absence de fondement métaphysique à sa morale: prêchant le retour à l'Évangile, il nie les dogmes de l'Église, mais ce faisant sa morale acquiert un caractère purement empirique. C'est la conséquence de son scepticisme théorique, qui nie toute connaissance hors de la conscience de soi et de ce qu'elle enseigne sur la vie, ce qui l'amène à condamner aussi bien les sciences, l'art, que les institutions politiques. Scepticisme qui est le corollaire «d'un idéalisme et d'un spiritualisme excessifs et étroits ».

Au terme d'une pareille analyse, Grot n'a évidemment pas d'autre choix que de s'en remettre à la solution médiane, la moins spectaculaire, qu'il évoquait en début d'article: reconnaissance d'un dualisme, recherche d'un nouveau compromis entre christianisme et paganisme, sur la «nouvelle base» de la modernité. Nietzsche est compris en dernière analyse comme une forme parmi les autres de l'un des deux pôles d'une opposition récurrente, celle de Socrate et de Démocrite, de Platon et d'Aristote, de l'idéalisme et du réalisme, et en définitive de la raison théorique et de la raison pratique de Kant. Grot cite alors la formule de Heine, que Nietzsche utilise également dans le premier paragraphe de Par-delà bien et mal: "Das ist eine alte Geschichte, doch bleibt sie immer neu» ("c'est une vieille histoire, mais il semble pourtant qu'elle vienne à peine de commencer»). L'originalité de la fin de l'article est d'interpréter le «pathos de la distance» comme un «sens de la perspective», qui serait précisément ce qui fait défaut à Tolstoï, et ce dont devra être doté le penseur qui réconciliera les deux points de vue qui s'affrontent. Grot semble entendre par là un sens des réalités historiques, qui interdit de déraciner les personnes de leur sol. Mais peut-être à des fins apologétiques, il réaffirme que le tolstoïsme n'est pas dangereux pour le «sol» lui-même, mais seulement pour la plante qui s'en arrache, réaffirmant ainsi son optimisme dans la rationalité et la cohérence du mouvement historique et sa certitude que tous les défenseurs du retour en arrière ne risquent pas de renverser la vapeur de la modernité.

Le deuxième article de réponse à Preobrajenski est donc au moins aussi critique que le premier. Nietzsche, à nouveau, est compris comme un symptôme typique du mal de l'époque, un penseur original mais enclin à l'exagération, cohérent dans ses refus, mais finalement impuissant. Ainsi Grot, en signalant que le surhomme n'est qu'un «rêve subjectif de Nietzsche, qu'il n'impose à personne, et qu'il ne fait que proposer à l'examen » rejoint Lopatine dans son estimation dépréciative de la part "positive» du nietzschéisme, et dans l'idée que sans moralité absolue, il n'y a aucune moralité qui tienne. Adoptant un critérium d'évaluation morale, Grot ne peut que condamner Nietzsche, dès lors qu'il l'assimile au primat de l'énergie physique sur le développement moral, ou plus exactement, dès lors qu'il lui 
attribue la thèse selon laquelle un progrès physique entraînera un progrès moral, alors qu'il défend le principe inverse.

\section{Astafiev}

On retrouve plusieurs des caractéristiques communes aux articles de Lopatine et Grot dans la troisième réponse, celle de Piotr Evguenievitch Astafiev ${ }^{4}$, intitulée: "Genèse de l'idéal moral d'un décadent ».

À nouveau, Nietzsche est déclaré conséquent dans l'exacte mesure où le commentateur utilise sa démonstration, c'est-à-dire en l'occurrence dans le travail de casse du relativisme moral, et la partie constructive de son enseignement n'est pas jugée digne que l'on s'y attarde, on l'écarte au contraire en faisant allusion à la folie de Nietzsche.

L'article d'Astafiev est sans doute celui des trois dont le ton est le plus hostile à Nietzsche, c'est également celui qui apporte le moins d'éléments à la discussion; bien qu'il mentionne Nietzsche contre Wagner, Astafiev n'en fait aucun emploi, et toutes ses critiques prennent pour base le travail de Preobrajenski. Il insiste d'ailleurs sur le fait que ce dernier n'est qu'un «simple exposé», ce qui a manifestement pour objectif de renvoyer toute la responsabilité des idées qu'il contient sur le compte de Nietzsche, ce en dépit des signes évidents de sympathie dans le texte de Preobrajenski. Saluer un article sur la critique de l'altruisme en parlant du "vrai sacrifice de soi» de l'auteur ne manque en tout cas pas de piquant.

La perspective dans laquelle s'inscrit Astafiev en début d'article est résolument passéiste, sinon réactionnaire: l'époque est jugée particulièrement riche en mauvais livres, et pauvre en œuvres «nées des principes et idéaux créés par la culture passée, prouvant sa vitalité, [...] et que jusqu'à une époque récente on admettait universellement». Mais Astafiev s'appuie sur une citation de Goethe (qui remonte en réalité à Pline l'Ancien) en guise de préambule à son étude: «Il n'y a pas de livre si mauvais qu'on ne puisse en apprendre quelque chose de bon». La distinction sur laquelle Astafiev construit son texte est celle entre morales conditionnelles et morale inconditionnelle. Il s'agit de montrer que Nietzsche a parfaitement réfuté les premières, mais que ses attaques ont laissé la seconde intacte, et que de surcroît sa propre entreprise de fondation s'autodétruit. Sous la catégorie de morales conditionnelles (c'est-à-dire dont le fondement est relatif), sont regroupés entre

48. Петр Евгеньевич Астафьев (1846-1893), professeur, a consacré ses travaux à combattre le rationalisme «relativiste» de l'idéalisme allemand, au nom d'une philosophie russe, spiritualiste et personnaliste, inspirée des slavophiles et de Leibniz. Il s'est également signalé par des travaux sur le psychisme féminin et ce qu'il appelle le «rythme psychique». 
autres «l'utilitarisme, la théorie de l'adaptation, l'évolutionnisme, la religion de l'humanité», auxquels il ajoute plus loin le pessimisme et l'altruisme; toutes ces doctrines se ramènent selon lui en dernière analyse au socialisme et à l'anarchisme, selon que la personne doit servir la société ou le contraire. Nietzsche est donc à nouveau rangé sous l'étiquette " anarchisme», assimilée au «bellum omnium contra omnes» de Hobbes. Le caractère spécifique des morales relatives est d'être des morales des fins (des buts). Ce à quoi Astafiev oppose une morale des principes, la seule valable, qui trouve son expression la plus parfaite dans le christianisme.

Astafiev ne distingue pas ce qui dans l'article de Preobrajenski est l'œuvre de Preobrajenski lui-même, ce qui l'amène à construire tout son argumentaire plutôt en réponse aux formalisations de son collègue qu'en réponse à Nietzsche lui-même. Deux thèses sont ainsi discutées, la première selon laquelle tant qu'aucune fin n'est posée, il n'y a pas davantage d'obligation morale (Aurore, $\$ 108$ ), la seconde selon laquelle pour juger des fins, il faut se placer en dehors de la morale (Le gai savoir, $\$ 380$ ), Preobrajenski ayant écrit "à l'extérieur et au-dessus», c'est de cette formule que se sert Astafiev pour proposer une réfutation par analogie avec la logique:

Que dirait-on d'un «penseur» qui nous assurerait sérieusement que pour juger sainement de la logique, il faut se placer à l'extérieur et au-dessus de la logique, renoncer à ses exigences et à ses lois? (p. 99)

On n'arrive de la sorte qu'à devenir immoral (ou illogique), pas du tout à poser ni à résoudre le moindre problème. L'analogie est fondée par le postulat que la morale tient aux «lois de notre esprit», par conséquent à des lois intérieures, (non à celles des objets extérieurs): impossible de sortir des frontières de la logique sans cesser de penser - la même chose vaut dès lors pour la morale et la volonté. Il ne saurait s'agir de créer les lois de ce qu'on étudie, mais seulement d'éclairer et de découvrir ce qu'Astafiev nomme «organisation spirituelle». Tout le mérite de Nietzsche est d'avoir montré que les fins supérieures qui prétendent ordonner et déterminer les systèmes de moralité ne sont pas elles-mêmes morales, sans quoi l'on aurait affaire à un cercle vicieux, puisque la moralité serait définie par la moralité de sa fin.

Qu'il existe néanmoins une loi morale, Astafiev l'affirme en prenant à témoin «toutes les doctrines morales, sauf celle de Nietzsche». Mais cette loi est présente à leur fondement soit de façon ouverte et clairement formulée, soit comme un postulat peu clair et demi-conscient. Le critère de la moralité est en fait «un principe de conformité formelle à la lo $i$ », c'est ce qui fait que chaque conscience exige que ses pensées s'accordent à un critère général, et qui est à l'origine de toutes les critiques et des distinctions entre vérité 
et mensonge, bien et mal, beauté et laideur. Cette exigence découle de ce que nous avons conscience de nous-même; aptes à objectiver nos pensées, nous pouvons les comparer avec d'autres et les juger. Bien que l'influence kantienne soit évidente, Astafiev ne considère l'impératif catégorique que comme la formulation «la plus abstraite», "purement formelle et logique», et l'oppose au commandement chrétien, «aime ton prochain comme toimême», "plus concret et plein». En effet:

La seule connaissance de la loi est encore insuffisante pour faire que la volonté eo ipso s'y soumette de bon gré. Je connais beaucoup de [...] lois de la nature, mais je ne m'incline devant elles que par nécessité, là où c'est inévitable; cependant partout où ce n'est que possible, je contourne ces lois, je les fais s'incliner devant moi, je les contrains à servir mes buts, ma volonté. (p. 108)

Et pour Astafiev, seule une loi donnée par «l'Être parfait» peut lier la volonté. L'obligation que la volonté s'impose à elle-même est ainsi déplacée sur le rapport entre volonté divine et volonté humaine. Malgré leur inconséquence formelle, l'utilitarisme et ses semblables sont également des formes dégradées de cette loi morale, qu'ils expriment dans des formules comme "chacun peut faire ce qui ne nuit pas aux autres». Refuser ce principe, si manifestement universel,

[...] c'est un pur nihilisme, dans lequel chaque raisonnement, chaque évaluation des pensées et des motifs se change en son contraire, en ce qui ne cherche sa justification en rien d'autre que soi : je veux ceci. Il n'y a plus de place ici pour aucune discussion, pour aucune preuve! (p. 106)

Or ce «je veux» constitue «l'essence même des opinions de Nietzsche», puisqu'il s'en remet pour l'imposition d'une fin morale à l'arbitraire de la volonté individuelle. Et Astafiev d'ajouter:

C'est dans ce culte de son propre arbitraire personnel comme loi suprême de l'être que se trouve également le noyau de cette démence qui s'est découverte plus tard, et dans laquelle a fini le malheureux Nietzsche, qui s'imaginait vers la fin de la vie qu'il était Dieu et le créateur du monde... (p. 106)

Où l'on retrouve textuellement les formules de l'avertissement apposé par la rédaction à l'article de Preobrajenski.

L'alternative nietzschéenne - soit la personne sert de moyen à la société, soit la société sert de moyen à la personne - méconnait l'idée de personne morale comme fin en soi qui ne saurait être utilisée comme un moyen, et se fonde tout entière sur le préjugé finalement inconsistant qu'il n'y a pas de morale absolue. C'est pourquoi Nietzsche comprend si mal la morale chrétienne et ne s'aperçoit pas qu'elle n'est pas fondée sur des buts, surtout 
pas extérieurs et utilitaires. Son tort est de croire que "toute soumission de la personne à une loi générale» équivaut à une dépersonnalisation et rend trivial (ce qui l'amène selon Astafiev à soutenir qu' "il ne doit pas y avoir de loi en général»): est-ce le cas du philosophe qui se soumet aux lois de la logique? Ou celui du compositeur qui se soumet aux lois de l'harmonie? Astafiev reconnaît que les morales altruistes, utilitaristes, et autres, produisent cet effet, et reprend même la notion de "chaînes" qui détruisent la force vitale.

Mais qui dans l'histoire a le plus œuvré à l'affranchissement de l'homme justement de ces chaînes dépersonnalisantes [...] ? - Le christianisme, bien sûr. (p. 110)

La conclusion de l'article, qui qualifie le nietzschéisme de «folie raisonnante de notre décadence», reprend les principaux éléments de l'argumentation:

Les opinions morales monstrueuses de Nietzsche sont instructives justement comme preuve convaincante et indirecte de ce que le fondement de la morale n'est pas extérieur (dans les buts), mais intérieur (dans les lois de l'esprit), n'est pas conditionnel et utilitaire, mais inconditionné et désintéressé. (p. 112)

On fait donc endosser à Nietzsche l'habit d'un Méphisto, qui travaille à son corps défendant au triomphe de la cause qu'il combat avec le plus d'acharnement - et à l'inverse de Grot, Astafiev n'en appelle pas à un quelconque travail de recherche à venir, puisque la combinaison de la loi morale chrétienne et du travail de sape nietzschéen fournit en définitive tout ce dont l'on pourrait avoir besoin en matière de morale.

Malgré leur opposition en ce qui concerne Nietzsche lui-même, on peut conclure à un certain consensus entre Preobrajenski et ses critiques: la «morale de l'altruisme» ne trouve pas parmi ces derniers de défenseur inconditionnel, et la raison en est sans doute que ces mots recouvrent en même temps la notion voisine, qui fonctionne comme un synonyme, mais ne se trouve pas dans le texte nietzschéen, de morale «sociale», autrement dit de morale ordonnée par la préoccupation du bien commun, du bien du peuple. Il semble que ce soit la raison pour laquelle la notion même d'altruisme ne fait pas chez les critiques l'objet d'une discussion approfondie, alors même qu'elle était le titre annoncé de l'article de Preobrajenski. Lopatine, Grot et Astafiev ont unanimement concentré leurs tirs sur les conceptions jugées les plus scandaleuses, en première ligne le surhomme exploitant les masses, sans réellement discuter les enjeux des passages d'Aurore qui occupent une 
place centrale chez Preobrajenski - des questions comme : «la compassion est-elle un gage de compréhension?», "est-il possible d'agir sans être mû en dernier ressort par l'égoïsme?», ont finalement peu été exploitées. Or la plus grande part de la dimension "positive» du nietzschéisme tel que l'expose Preobrajenski est postérieure à Aurore. La question n'est pas réellement abordée de savoir jusqu'où toutes les thèses présentées comme un seul bloc sont solidaires ou au contraire jusqu'où la séparation tranchée entre sape et fondation est justifiée - ce qui conditionne pourtant la thèse tout de même discutable selon laquelle la part négative de Nietzsche serait globalement valide, et sa part positive, imputable à sa folie, pas même digne d'une discussion approfondie.

Antoine MULLER

ENS Lyon 